\title{
De la nature humaine au sujet transcendantal : Comment l'activité libre du moi et sa responsabilité à l'égard de la raison théorique et pratique s'étayent sur la dynamique propre à la sphère passive
}

\author{
Par BRUNO LECLERCQ \\ Université de Liège
}

Résumé Il est possible de proposer une lecture génétique de l'œuvre de Husserl tout entière axée sur la tension - et la tentative de conciliation - entre exigences rationalistes et explications empiristes ; une fois le rationalisme radicalement assuré par les thèses fortes marquant le double tournant antipsychologiste puis transcendantal, les préoccupations génétiques initiales héritées de la tradition empiriste peuvent retrouver toute leur pertinence pour autant qu'elles soient rapportées à la sphère de la passivité, qui «motive » l'activité subjective sans la déterminer. Tout à la fois distincte de la causalité naturelle et de la justification rationnelle, cette "motivation » joue un rôle crucial dans le dispositif théorique. Reste toutefois à rendre compte de l'économie psychique propre à cette subjectivité tout à la fois passive et active. Inspirées de Husserl mais aussi de Freud, de belles analyses de Rudolf Bernet montrent ce qu'il en est dans la sphère volitive. D'origine naturelle (biologique) mais de nature psychique (et, en ce sens au moins, « conscientes »), les pulsions enjoignent le moi de les satisfaire. Pour s'exécuter au mieux, le moi, qui est aussi redevable à l'égard du surmoi et du principe de réalité (c'est-à-dire, en gros, des exigences de la raison pratique et de la raison théorique), s'efforce de trouver une solution à l'équation des contraintes auxquelles il est soumis. Il en vient alors à donner son aval à ces pulsions sous la forme de désirs et de souhaits intentionnels qu'il juge acceptables (et susceptibles de les satisfaire sur le long terme), ou au contraire à inhiber ces pulsions sous les modalités de leur investissement spontané. Dans ce cas, toutefois, c'est encore dans des pulsions (pulsions en sens contraire, pulsions d'autoconservation, etc.) que le moi 
trouve la force nécessaire à s'opposer. Prenant au sérieux la suggestion de Bernet selon laquelle les sensations ont elles-mêmes un caractère pulsionnel, nous nous efforçons ici d'évaluer la transposition possible de ses analyses dans la sphère judicative. Dans cette perspective, perceptions et jugements intentionnels constituent la meilleure réponse qu'apporte le moi actif à la pression des sensations et à leur modalisation spontanée sous forme de synthèses passives. Soucieux de ses intérêts à long terme, le moi s'avère capable de rejeter certaines données sensorielles et de s'opposer à certaines tendances psychiques qui se font pourtant pressantes.

En phénoménologie, nos préoccupations, qui sont celles d'un théoricien de la connaissance, se portent généralement en priorité sur le pôle noématique des phénomènes - c'est-à-dire sur des contenus d'actes de représentation et de jugement de nature idéale, ainsi que sur les rapports de compatibilité et de conséquence entre de tels contenus idéaux, rapports qui fondent les relations de justification entre les actes psychiques correspondants - et s'orientent seulement dans un second temps sur le pôle noétique des phénomènes - c'est-àdire sur les actes intentionnels qui ont présidé à la constitution et à la stabilisation de ces contenus et rapports idéaux. Un peu exceptionnellement, il s'agira toutefois ici de nous livrer à un pur exercice de psychologie descriptive, voire même de nous aventurer dans ce que les Brentaniens qualifieraient sans doute de psychologie génétique ou explicative, dans la mesure où nous nous efforcerons d'étudier l'énergétique psychique sous-jacente à l'émergence même des actes intentionnels caractéristiques de la rationalité cognitive et de la liberté qu'elle présuppose.

\section{La théorie husserlienne de la motivation}

Pour comprendre le niveau où se tiendront nos analyses, nous nous permettrons tout d'abord de resituer les présentes investigations à l'égard d'un certain nombre de nos recherches antérieures. Tout d'abord, notre lecture générale de la phénoménologie husserlienne, telle qu'explicitée dans notre ouvrage sur les Fondements logiques et phénoménologiques de la rationalité mathématique ${ }^{1}$,

${ }^{1}$ B. Leclercq, Fondements logiques et phénoménologiques de la rationalité mathématique. Contributions husserliennes au débat sur la "crise des fondements », Paris, Vrin, 2015. 
mais aussi de manière plus synthétique dans quelques articles ${ }^{1}$, consiste à y voir une longue entreprise de conciliation entre des tendances empiristes et des tendances rationalistes, entreprise qui, dans l'itinéraire philosophique de Husserl, passe, on le sait, par un certain nombre de tournants théoriques qui s'efforcent chaque fois de corriger ou du moins de nuancer les résultats antérieurs.

Héritier de Brentano et Stumpf, Husserl débute ses investigations philosophiques par des considérations résolument empiristes, qui s'efforcent d'éclairer toute représentation, y compris une représentation mathématique comme celle d'un nombre, par la reconstitution de sa genèse à partir de diverses expériences élémentaires (quoique pas forcément atomiques) et moyennant certains actes psychiques de colligation, d'abstraction, etc., qui permettent de dégager des contenus de représentation qui n'étaient pas directement donnés dans l'expérience ${ }^{2}$.

Théorisé dans les « Prolégomènes à la logique pure », premier volume des Recherches logiques, le virage antipsychologiste de la phénoménologie dénoncera la naïveté de ce premier projet au nom de l'objectivisme sémantique bolzanien, c'est-à-dire en faisant valoir l'idéalité des contenus de représentation, laquelle contraste avec la réalité des actes psychiques de représentation qui visent ces contenus, et l'irréductibilité des liens logiques qui rapportent ces contenus les uns aux autres aux liens réels, notamment causaux, qui rapportent les actes psychiques les uns aux autres ; la légalité logique idéale qui régit les contenus ne peut en aucun cas s'expliquer par la légalité psychique réelle qui régit les actes, de sorte que la stratégie empiriste d'élucidation génétique est inappropriée $e^{3}$.

Dans la mesure, toutefois, où il convient bien de rendre compte de la manière dont ces contenus et leurs rapports idéaux sont visés et donnés dans les actes intentionnels de sujets pensants et connaissants, c'est tout de même à

\footnotetext{
${ }^{1}$ B. Leclercq, «Phénoménologie et pragmatisme : y a-t-il rupture ou continuité entre attitudes théoriques et attitudes pratiques ?» dans Bulletin d'analyse phénoménologique vol. 4 n³ (2008), p. 81-123 ; «Les Motifs entre activité et passivité de la conscience. Entre l'ordre des causes et celui des raisons » dans M. Gyemant et D. Popa [dir.], Approches phénoménologiques de l'inconscient, Hildesheim, Olms, 2015, p. 37-50 ; «Lois régissant les phénomènes : légalités noématiques, noétiques et hylétiques » dans Bulletin d'analyse phénoménologique (numéro spécial Entre phénoménologie et psychologie : la passivité) vol. $8 \mathrm{n}^{\circ} 1$ (2012), p. 121-139 ; B. Leclercq et S. Richard, «Edmund Husserl », rubrique (niveau académique) de l'Encyclopédie Philosophique, mise en ligne en 2016.

${ }^{2}$ E. Husserl, Philosophie de l'arithmétique, trad. fr. J. English, Paris, PUF, 1972.

${ }^{3}$ E. Husserl, « Prolégomènes à la logique pure », vol. I des Recherches logiques, trad. fr. H. Elie, A. Kelkel et R. Scherer, Paris, PUF, 1959-1963.
} 
des considérations de psychologie descriptive (désormais qualifiée de «phénoménologie ») que Husserl a consacré l'essentiel des six Recherches, ce qui lui valut d'ailleurs de nombreuses accusations de retombée dans le psychologisme de la part de certains lecteurs enthousiastes des « Prolégomènes », tels Paul Natorp ${ }^{1}$, Alexius Meinong ${ }^{2}$ ou Louis Couturat ${ }^{3}$. Et c'est pourquoi il convenait qu'il clarifie le statut même de cette psychologie descriptive ou " phénoménologie ».

S'il s'agit de fonder une théorie de la connaissance, il est clair qu'il ne suffit pas, d'une part, de reconnaitre l'idéalité de rapports logiques sur le plan du contenu et, d'autre part, de se livrer à l'étude empirique des actes psychiques à travers lesquels des sujets réels pensent ces rapports; comme l'avaient souligné les «Prolégomènes à la logique pure » en distinguant psychologie empirique, logique idéale et logique normative, les lois logiques idéales qui prévalent au niveau des contenus impliquent des normes pour les actes de représentation et de jugement qui prétendraient se conformer à elles. Or, seul un sujet transcendantal libre, et non un être naturel soumis à des lois psychiques causales, peut répondre d'une telle normativité ; comme c'est aussi le cas sur le plan pratique à l'égard des normes morales, être responsable suppose d'être libre : « Pour devoir, il faut pouvoir ». Et c'est là la clé de compréhension du tournant transcendantal qu'imprime Husserl à la phénoménologie après les Recherches logiques ; en théorisant, non pas le fonctionnement naturel du psychisme humain, mais la structure intentionnelle d'une subjectivité libre susceptible de se conformer aux exigences logiques, Husserl se donne les moyens d'une théorie de la connaissance rationaliste comparable à celle de Natorp puisqu'elle combine les même éléments de platonisme sur le plan objectif (noématique) et de kantisme sur le plan subjectif (noétique) ${ }^{4}$.

Restera alors à réintroduire la vérité de l'empirisme au sein de cette théorie de la connaissance rationaliste, à savoir que, quoique libre, l'activité noétique du sujet transcendantal n'est pas arbitraire, mais sans cesse motivée par l'expérience. En particulier, les synthèses qu'opère activement le sujet

\footnotetext{
${ }^{1}$ P. Natorp, « Compte rendu des "Prolégomènes à la logique pure" ", cité dans « Esquisse d'une préface aux Recherches logiques » (1903) dans Articles sur la logique, trad. J. English, Paris, PUF, 1975, p. 361.

2 A. Meinong, Über Gegenstandstheorie, Leipzig, Barth, 1904, § 7 ; trad. fr. M. de Launay et J.-F. Courtine, La Théorie de l'objet, Paris, Vrin, 1999, p. 84-86.

${ }^{3}$ L. Couturat, Lettre du 7 novembre 1904 dans E. Husserl, K. Schuhmann et E. Schuhmann [dir.], Briefwechsel, Vol. VI : « Philosophenbriefe », Dordrecht, Kluwer Academic Publishers, 1993, p. 33-35.

${ }^{4}$ E. Husserl, Idées directrices pour une phénoménologie I, trad. fr. P. Ricœur, Paris, Gallimard, 1950.
} 
transcendantal - en organisant les données de la sensation, en les animant intentionnellement et en leur donnant sens - ne sont pas entièrement laissées à son libre arbitre, mais elles lui sont en permanence suggérées par des synthèses passives qui s'effectuent au sein même du donné expérientiel ${ }^{1}$ : Gestalten, fusionnements, harmonies et autres associations passives, tous ces éléments qu'ont mis en évidence les empiristes de la fin du XIX ${ }^{\mathrm{e}}$ siècle, notamment Stumpf ${ }^{2}$, contre l'idée, commune aux empiristes britanniques et aux rationalistes, selon laquelle l'expérience serait une suite discontinue de données atomiques que devrait alors organiser le psychisme humain (par ses principes naturels d'association sous l'effet de l'habitude) ou le sujet transcendantal (par ses principes rationnels a priori).

La distinction même du niveau des synthèses passives, qui s'effectuent à même le donné et échappent à la maîtrise du sujet transcendantal, et des synthèses actives, qui sont activement opérées par le sujet transcendantal rationnel, permet à Husserl de se réapproprier les questions génétiques propres à l'empirisme sans plus prendre le risque du psychologisme et sans renoncer au rationalisme; dans son activité synthétique et noétique, le sujet transcendantal est motivé, mais pas causalement déterminé, par ce que suggère le donné ; en être responsable à l'égard de la raison, il peut ou non assumer activement les synthèses passives qui se proposent en permanence à lui, c'est-à-dire qu'il peut effectuer des vérifications à l'égard des synthèses qui lui paraîtraient mal assurées, mais aussi s'efforcer de concilier l'ensemble des synthèses suggérées en un tout cohérent, ce qui suppose sans doute de rejeter in fine certaines suggestions initiales et d'en accepter d'autres. La passivité sensible propose, mais le sujet actif dispose ; loin d'être naturellement déterminé par les processus psychiques qui pré-organisent son expérience, le sujet transcendantal est certes motivé par ces synthèses passives, de sorte qu'il n'a pas à les inventer arbitrairement, mais il conserve à leur égard toute sa liberté de sujet responsable à l'égard de la raison. Avec la "motivation » des synthèses actives par les synthèses passives, justice est rendue à l'empirisme sans que ne soit plus mis en danger le rationalisme.

Au-delà de cette relecture de l'itinéraire philosophique de Husserl, notre intérêt propre pour la problématique de la motivation tient évidemment dans le fait que ce cadre théorique husserlien permet effectivement de dessiner une théorie de la connaissance tout à la fois rationaliste et empiriste, et par là de

${ }^{1}$ E. Husserl, Expérience et Jugement, trad. fr. D. Souche-Dagues, Paris, PUF, 1970 ; De la synthèse passive, trad. fr. B. Bégout et J. Kessler, Paris, Millon, 1998.

${ }^{2}$ C. Stumpf, Über den psychologischen Ursprung der Raumvorstellung, Hirzel, Leipzig, 1873 ; Tonpsychologie, Hirzel, Leipzig, 1883, 1890. 
répondre au dilemme posé par un grand nombre d'épistémologues contemporains dans la foulée notamment de Wilfried Sellars ${ }^{1}$. Selon Sellars, on le sait, l'expérience ne peut, en tant que telle, fournir de justification rationnelle à nos jugements de connaissance car la justification rationnelle de jugements ne peut être fournie que par d'autres jugements. Dès lors, de deux choses l'une : soit l'expérience doit être considérée comme elle-même déjà judicative et donc de nature conceptuelle et propositionnelle ; soit, faute d'être elle-même déjà judicative, l'expérience échappe à la sphère des raisons et au mieux joue un rôle dans l'ordre des causes, c'est-à-dire qu'elle intervient dans la genèse causale des croyances mais qu'elle ne participe pas de leur justification rationnelle. On reconnaît là, sous sa double forme, la fameuse critique du « mythe du donné ».

Pour sa part, Husserl, en empiriste, entend bien reconnaître un rôle épistémique et non simplement psychogénétique à l'expérience antéprédicative. Or, c'est précisément ce qu'il peut faire grâce à la notion de " motivation », laquelle ne caractérise ni un pur lien de détermination causale ni un pur lien de justification logique d'ordre propositionnel ; il s'agit en fait d'une justification non logique, qui fournit des " motifs », c'est-à-dire des données qui pèsent en faveur d'une prise de position judicative sans pour autant l'impliquer logiquement. Et c'est ce qui explique que le sujet puisse y reconnaître des suggestions de synthèse qui ne contraignent pas complètement son activité rationnelle ; le sujet reste libre d'écarter certains motifs au nom d'autres motifs de force supérieure plaidant en sens contraire. Située (comme nous l'avons montré ailleurs ${ }^{2}$ ) entre l'ordre des causes et celui des raisons, la motivation rend au donné son rôle épistémique.

\section{Les soubassements énergétiques de la motivation}

Ainsi envisagée depuis les préoccupations de la théorie de la connaissance, la doctrine de la motivation est en fait encore incomplète dans la mesure où elle énonce essentiellement ce que présuppose la connaissance empirique et décrit

\footnotetext{
${ }^{1}$ W. Sellars, « Empiricism and the Philosophy of Mind » dans Science, Perception and Reality, London, Routledge and Kegan Paul, 1963, p. 127-196 ; cf. aussi D. Davidson, «A Coherence Theory of Truth and Knowledge » dans A.R. Malachowski [dir.], Reading Rorty, Oxford, Blackwell, 1990, p. 120-138.

2 B. Leclercq, «Les Motifs... », art. cit. ; «Le Tournant linguistique et son contrevirage phénoménologique » dans Études philosophiques (numéro spécial « La méthode phénoménologique aujourd'hui ») (2012/1), p. 7-26 ; « Le Mythe de la donation de sens ; motivation de la noèse par le donné », à paraître dans D. Pradelle et P.-J. Renaudie [dir.], Intentionnalité, sens, antipsychologisme. Hommage à Robert Brisart.
} 
alors l'activité mais aussi la passivité psychique d'après le rôle qu'elle doit pouvoir jouer dans ce processus de justification prélogique. On a d'ailleurs souvent reproché à Husserl d'avoir essentiellement conçu la passivité d'après les actes de connaissance qu'elle prépare et donc d'un point de vue qui est déjà celui de l'activité judicative ; les synthèses passives, mais aussi les modalités doxiques d'adhésion subjective à ces synthèses — croyance primaire, mise en doute suscitée par un conflit d'évidences, croyance réaffirmée après résolution de ce conflit, etc. - , sont en effet clairement envisagées et décrites d'après les synthèses actives et les prises de position rationnelles qui en feront de véritables actes de connaissance ${ }^{1}$. Or, il est sans doute possible, et certains textes de Husserl vont en ce sens, de décrire cette sphère de la passivité du strict point de vue de la psychologie descriptive, c'est-à-dire de décrire le fonctionnement psychique à l'œuvre dans ce qui n'est pas encore une activité subjective au sens propre. Avant même les actes intentionnels, l'esprit est fait d'un certain nombre de tendances voire de forces, lesquelles constituent le fondement naturel des synthèses passives mais aussi de l'émergence, en leur sein, d'une subjectivité proprement active. Dans la mesure où il s'agit de processus naturels qui participent de la genèse de la subjectivité, on sort là clairement du cadre de la phénoménologie transcendantale et même de celui de la seule psychologie descriptive au sens brentanien ${ }^{2}$ puisqu'on se préoccupe également d'une " dynamique psychique » ou d'une " énergétique psychique » qui relève de la psychologie génétique ou explicative et ne semble pas avoir de rôle à jouer en théorie de la connaissance (sous peine de retombée dans le psychologisme) quoiqu'elle constitue un complément intéressant de celle-ci dans une théorie unifiée de l'esprit.

Plutôt que de solliciter (comme nous l'avons fait ailleurs) les considérations explicites de Husserl en la matière, nous nous appuierons ici sur les très belles analyses fournies par Rudolf Bernet pour la sphère affective et volitive à partir de considérations aristotéliciennes, leibniziennes, schopenhaueriennes et bien sûr freudiennes relues phénoménologiquement. Sur une de ses suggestions, qui envisage de traiter la sensation sous sa dimension pulsionnelle, nous nous efforcerons de voir ce qui, de ses analyses dans la sphère affective et volitive, peut être transposé dans la sphère cognitive. Ce qui intéresse en effet Bernet dans son beau livre Force - pulsion - désir. Une autre philosophie

${ }^{1}$ Cf. E. Husserl, Expérience et Jugement, op. cit. ; De la synthèse passive, op. cit.

${ }^{2}$ Explicitement revendiquée par Brentano dans Meine letzten Wünsche für Österreich, Stuttgart, Cotta, 1895, p. 34-35, cette distinction est en fait déjà sous-jacente à l'ensemble même de la démarche qui était effectuée en 1874 dans la Psychologie du point de vue empirique, trad. fr. M. de Gandillac, Paris, Vrin, 2008.

162

Bull. anal. phén. XVI 2 (2020)

https://popups.uliege.be/1782-2041/ @ 2020 ULiège BAP 
de la psychanalyse ${ }^{1}$, c'est l'énergétique psychique qui anime l'esprit d'un certain nombre de forces diversement orientées, lesquelles rendent possible l'émergence de pulsions, de désirs orientés puis, sur leur base, de souhaits proprement intentionnels. Il conviendrait donc de savoir si une même structure peut être retrouvée dans la sphère cognitive, c'est-à-dire si des perceptions proprement intentionnelles émergent de la même manière de ce qui est d'abord un jeu de forces psychiques et même organiques.

Le grand intérêt de l'ouvrage de Bernet, c'est - en s'appuyant sur cet autre auditeur de Brentano que fut Sigmund Freud - de reconduire la psychologie intentionnelle des émotions et des désirs aux affects, aux pulsions et même aux forces biologiques qui les sous-tendent. Bernet, qui est d'abord et avant tout phénoménologue, est parfaitement conscient du caractère périlleux de cet exercice, qui s'apparente à une naturalisation de l'esprit et prend le risque du biologisme. Ce que Bernet veut toutefois mettre en évidence, c'est le potentiel intentionnel que comportent déjà forces et pulsions : quoiqu'encore dépourvus de toute valeur symbolique, ces éléments de la dynamique psychophysique sont vecteurs de différences (intensives, qualitatives, etc.) qui revêtent de l'importance et par là même déjà une certaine signification ${ }^{2}$. On peut alors montrer comment, de ces processus psychophysiques, peuvent progressivement jaillir des affects et des pulsions dotés de certaines orientations puis, sur leur base, des actes intentionnels et même une certaine capacité d'opérer des choix. C'est donc à une certaine genèse de la subjectivité, dotée de liberté et de rationalité, à partir de ce qui apparaît d'abord comme une pure et simple dynamique biologique que se livre Bernet. À cet égard, il s'agit moins de naturaliser la conscience intentionnelle que de montrer, en sens inverse, que, dans la nature même de l'homme - corps et esprit —, se trouvent déjà en puissance tous les ferments de ce qui fera la spécificité de la conscience intentionnelle :

Rien ne préserve mieux l'énergétique psychanalytique d'une retombée dans le biologisme mécaniste qu'une réflexion philosophique concernant la tension pulsionnelle comme intensité, et cette intensité comme différence qualitative ${ }^{3}$.

\footnotetext{
${ }^{1}$ R. Bernet, Force - pulsion - désir. Une autre philosophie de la psychanalyse, Paris, Vrin, 2013.

${ }^{2}$ R. Bernet, Force - pulsion - désir, op. cit., p. 16.

${ }^{3}$ Ibid.
} 
C'est avec la notion aristotélicienne de dunamis que Bernet, s'appuyant sur Heidegger, entame cette réflexion philosophique. Tandis qu'hommes et animaux sont dotés d'une dunamis vitale ${ }^{1}$, qui les met en mouvement dans la mesure où elle tend à s'accomplir en energeia, l'homme semble quant à lui pourvu d'une dunamis meta logou dont la caractéristique principale tient dans une certaine retenue à l'égard de l'actualisation de la dunamis (« l'exercice du pouvoir $»)^{2} ;$ l'homme semble en mesure de postposer, voire même de renoncer à, l'actualisation de certaines de ses tendances vitales (tendances à aller vers et tendances à éviter ${ }^{3}$ ), et c'est dans cette retenue, corrélative d'une obligation de choisir entre actualiser ou non les tendances, que tient la première forme du logos. Mais cela n'est possible que parce que les tendances reposent elles-mêmes sur un jeu complexe de forces actives et passives qui tout à la fois crée des tensions et ouvre un espace de possibles. La matière est en effet le lieu de forces passives (dunamis tou pathein), qui produisent tout à la fois une capacité à supporter certaines formes et une capacité à résister à certaines formes ${ }^{4}$. Les forces actives (dunamis tou poiein), quant à elles, se divisent en capacité d'imposer des formes et capacité de ne pas imposer ${ }^{5}$; la seconde est l'adunamia, une non-force ou privation (steresis) de force, qui peut être due à la fatigue, à la prostration ou à d'autres causes internes d'empêchement ${ }^{6}$. Cette possibilité qu'ont les forces actives de s'épuiser ou de connaître des " pannes » explique par le fait que la dunamis n'implique pas toujours l'energeia. C'est là que s'institue un peu de jeu dans la dynamique naturelle, jeu qui ouvre des possibles et par là même l'éventualité de « choix ».

Entre la dunamis et l'energeia correspondante, il n'y a, en principe, rien d'autre qu'un passage à l'actualisation ; la dunamis contient déjà en puissance tout ce que l'energeia contiendra en acte. Mais le fait que, notamment chez l'homme, la dunamis ne s'actualise pas systématiquement, qu'on en reste parfois, ou pendant un certain temps, au stade de la dunamis, ne s'explique pas seulement par le fait que des forces extérieures contrecarrent la force active de la dunamis et sa tendance naturelle à s'actualiser; il se peut aussi que cette force active soit contrecarrée par des forces inhibitrices internes à la dunamis,

\footnotetext{
${ }^{1}$ Ibid., p. 27.

${ }^{2}$ Ibid., p. 44.

${ }^{3}$ Ibid., p. 47.

${ }^{4}$ Ibid., p. 50.

${ }^{5}$ Ibid., p. 51.

${ }^{6}$ Ibid., p. 56.
} 
qu'il s'agisse de l'adunamia, privation de force, ou d'une force passive de résistance due à la matière sur laquelle s'exerce la dunamis ${ }^{1}$. L'actualisation de la dunamis suppose alors que soient surmontées ces inhibitions, que la dunamis soit activement désinhibée, ce qui suppose donc une force active supplémentaire à la seule dunamis initiale. C'est donc dans cette possibilité de désinhiber ou non la dunamis que se situerait la première opportunité pour le logos ; tel serait le principe de la dunamis meta logou ${ }^{2}$.

C'est, selon Bernet, à Leibniz qu'il revient d'intégrer ces éléments dans une véritable théorie de la subjectivité. Pour Leibniz, un corps matériel est doté de forces actives responsables de son mouvement et de forces passives qui expliquent sa résistance à certaines forces externes - on voit là l'idée leibnizienne de l'inertie - de sorte que les mouvements d'un corps matériel s'expliquent entièrement par ses propres forces internes ${ }^{3}$. De l'extérieur, et notamment de ses contacts et chocs avec d'autres corps - analogiquement qualifiés de « perceptions »-, un corps ne reçoit pas directement du mouvement mais seulement la contrainte de réorganiser ses propres forces internes, réorganisation qui peut alors résulter en une modification de son mouvement. Le jeu des forces actives et passives propres à un corps implique une véritable dynamique dans la mesure où les forces actives — analogiquement qualifiées d' " appétitions $»^{4}$ - pressent à se réaliser - et sont donc plus conformes à ce que Bergson appellera « virtualités $»^{5}$ qu'à de simples potentialités - mais se heurtent à la résistance de forces passives ${ }^{6}$. Selon l'organisation des forces en présence, ces dernières peuvent triompher ou être vaincues, mais elles ne disparaissent de toutes façons jamais, le corps restant en permanence soumis à une tension entre les forces internes qui le constituent; même l'état de repos est un état dynamique résultant d'une tension entre forces antagonistes plutôt qu'un état statique $^{7}$. Une monade est alors un tel jeu de forces envisagé en tant qu'il vise à devenir substance et unifier ses forces constitutives ${ }^{8}$. Ensemble unifié d'appétitions et de perceptions, elle est alors un point de vue singulier sur le monde, point de vue qui se modifie au gré des mouvements du corps. Résultante d'une puissante dynamique, l'unité monadique, toutefois, n'est jamais parfaitement

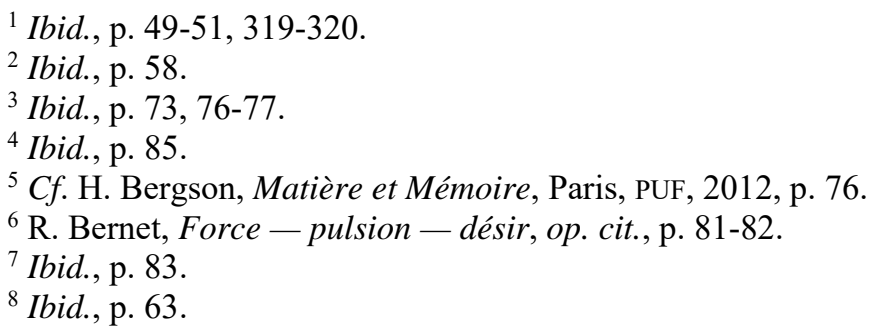


acquise $^{1}$, et ses tensions internes jamais parfaitement résorbées. C'est pourquoi, par exemple, l'homme n'est jamais parfaitement à son aise, mais toujours inquiet - comme le balancier d'une horloge (Unruhe $)^{2}$ — et, en raison de cette instabilité, sans cesse habité d'un manque ou d'un désir ontologique confus ${ }^{3}$, lequel est au fondement de toutes ses pulsions et de tous ses désirs psychologiques ultérieurs ${ }^{4}$.

Pour sa part, dit toujours Bernet, Schopenhauer fait comprendre que les désirs de la volonté rationnelle ne sont pas de nature distincte des pulsions animales. Dans les deux cas, il s'agit de forces pressantes mais virtuelles qui ont besoin d'excitations pour s'actualiser en se libérant de leurs entraves ${ }^{5}$. Mais, alors que des excitations physiques suffisent à désinhiber les premières, les désirs de ma volonté rationnelle se caractérisent uniquement comme tels par le fait qu'ils sont désinhibés par des " motifs », c'est-à-dire des représentations de circonstances futures tenues pour souhaitables ${ }^{6}$. C'est seulement l'existence ou non de telles représentations qui fait la différence entre des pulsions animales et des désirs rationnels ${ }^{7}$. Dans les deux cas, c'est bien la volonté — conçue dans un sens large — qu'il s'agit de satisfaire ${ }^{8}$; si, faute d'être déclenchée par des motifs, l'activation des pulsions animales est dite « involontaire », le fait qu'elle donne lieu à un sentiment de plaisir témoigne bien qu'elle est déjà au service de la volonté ${ }^{9}$. Dans les deux cas, il s'agit essentiellement de permettre à une force pressante de s'actualiser, de sorte que les motifs rationnels ne sont en fait, comme les excitations physiques, que des occasions de passage de la puissance à l'acte ${ }^{10}$. C'est pourquoi être au service de la raison n'empêche pas d'être au service de la volonté. Comme le dit Bernet :

\footnotetext{
${ }^{1}$ Ibid., p. 112.

${ }^{2}$ Ibid., p. 98.

${ }^{3}$ Ibid., p. 99.

${ }^{4}$ Ibid., p. 102.

${ }^{5}$ Ibid., p. 152.

${ }^{6}$ Ibid., p. 156-157.

${ }^{7}$ Ibid., p. 154.

${ }^{8}$ Ibid., p. 137-138.

${ }^{9}$ Ibid., p. 139-140.

${ }^{10}$ Ibid., p. 144.
} 
Il s'ensuit que l'autodétermination du sujet rationnel de l'action et donc aussi sa liberté ne sont jamais totales, et que l'homme qui agit d'une manière raisonnable reste toujours tributaire de son caractère empirique. Même en agissant selon les principes de la raison, l'homme continue à servir la Volonté ${ }^{1}$.

La raison humaine n'est au fond qu'une ruse de la volonté ; pour mieux se satisfaire, la volonté développe l'intelligence, laquelle déploie des représentations susceptibles de constituer des motifs désinhibant les forces pressantes ${ }^{2}$. Intéressant en ce qu'il prépare Freud, Schopenhauer développe même, selon Bernet, toute une théorie du refoulement dans la mesure où la volonté parasite la conscience en en écartant certains motifs qui l'attirent secrètement mais qu'elle ne veut pas reconnaître explicitement ${ }^{3}$. Mais Schopenhauer prépare aussi la théorie freudienne de la sublimation par une réflexion sur la manière dont l'expérience esthétique du sublime est celle d'un dépassement des sentiments hostiles à la volonté - peur, terreur, angoisse, etc. - provoqués par l'image effrayante, au profit de la contemplation désintéressée (désaffectée) des idées platoniciennes qui s'instancient dans cette expérience ; en tenant exceptionnellement à distance sa propre volonté, le sujet pulsionnel se fait alors sujet transcendantal ${ }^{4}$.

La théorie freudienne des pulsions soumises au principe de plaisir principe assez complexe qui ne vise pas nécessairement la détente totale, mais peut au contraire se satisfaire dans un rythme alternatif d'augmentation et de diminution de la tension ${ }^{5}$, et en sens inverse trouver dans l'apaisement une source de frustration et de nouvelle excitation ${ }^{6}$ — est le paradigme même de la théorie de l'activité psychique qui se joue dans un intermédiaire entre mécanismes purement naturels et gestion proprement subjective. Bien que ses modèles théoriques soient inspirés des sciences naturelles (mécanique, électricité, thermodynamique, etc.), Freud, selon Bernet, n'est pas naturaliste et il reconnaît une certaine spécificité à l'activité psychique, laquelle n'est toutefois pas entièrement le fait d'une subjectivité rationnelle ${ }^{7}$. Les pulsions ne sont, pour Freud, pas des tensions organiques, mais les représentants psychiques de ces tensions ; à cet égard, ce qui s'y joue, dit Bernet, est essentiellement de l'autoaffection puisque l'esprit qui agit sous l'effet des pulsions ne subit l'action que

${ }^{1}$ Ibid., p. 164.

${ }^{2}$ Ibid., p. 166-167.

${ }^{3}$ Ibid., p. 171-174.

${ }^{4}$ Ibid., p. 397-398.

${ }^{5}$ Ibid., p. 193.

${ }^{6}$ Ibid., p. 197, 206, 231.

${ }^{7}$ Ibid., p. 190-191. 
d'éléments déjà psychiques, donc internes ${ }^{1}$. Les éventuelles stimulations externes qui « déclenchent » ces pulsions n'en constituent pas les causes mais seulement l'occasion. Cela explique d'ailleurs, pour Bernet, l'extraordinaire plasticité des pulsions, et en particulier de la pulsion sexuelle. Dans la mesure où ce qui compte est l'économie psychique de la tension libidinale et sa satisfaction, la source organique des pulsions, mais aussi l'objet de leur satisfaction, et le but même de cette satisfaction sont en effet accidentels du point de vue du principe du plaisir. Tel ou tel objet, dit Freud, n'est qu'un moyen occasionnel d'atteindre la satisfaction libidinale ${ }^{2}$ — pour Lacan, on peut même dire que tout objet n'est jamais qu'un substitut pour l'objet, par essence manquant, de la pleine satisfaction ${ }^{3}$. Et l'apaisement organique n'est pas forcément synonyme de satisfaction psychique. C'est pourquoi sont possibles tous les phénomènes d'étayage de la pulsion à partir de sources organiques diverses ${ }^{4}$ et de détournement de la pulsion vers d'autres objets ou d'autres buts. Bernet se livre notamment à de belles analyses sur les processus psychiques de la répétition pulsionnelle, qui, loin de permettre l'apaisement organique, semblent finalement se relancer sans cesse en se prenant eux-mêmes pour objet et pour but.

Freud est aussi penseur de l'émergence du moi dans l'énergétique pulsionnelle. La seconde topique freudienne prévoit en effet un conflit des instances psychiques du fait que la conscience se mobilise énergiquement contre tout désir qui mettrait en péril sa cohérence ${ }^{5}$. On reconnaît là l'idée leibnizienne d'unification monadique, et, en particulier, cette idée que le moi doit concilier les exigences du ça avec celles du surmoi - lequel est partagé entre un moi idéal (représentant des normes internes) et un idéal du moi (représentant des normes externes $)^{6}-$, mais aussi avec celles de la réalité 7 . Or, dans la perspective énergétique dessinée jusqu'ici, on peut se demander d'où jaillissent le moi et le surmoi et d'où ils tiennent une volonté et une énergie capables de s'opposer aux forces pulsionnelles du ça. La solution freudienne à cette interrogation est au fond schopenhauerienne : moi et surmoi tiennent euxmêmes toute leur énergie du ça, lequel ne développe ses instances apparemment antagonistes que pour mieux servir ses intérêts ${ }^{8}$. Le moi a en effet pour

\footnotetext{
${ }^{1}$ Ibid., p. 196-197, 228-229.

2 Ibid., p. 235.

${ }^{3}$ Ibid., p. 236.

${ }^{4}$ Ibid., p. 202-203.

${ }^{5}$ Ibid., p. 343.

${ }^{6}$ Ibid., p. 366-367.

${ }^{7}$ Ibid., p. 357.

${ }^{8}$ Ibid., p. 360-361.
} 
principale fonction de freiner la fougue du ça... de manière à lui permettre de mieux réaliser ses désirs à long terme ${ }^{1}$, et ce en résolvant les conflits entre désirs incompatibles, entre désirs et valeurs (désirs de moralité) ou entre désirs et réalité. Le moi n'est à cet égard qu'une instance dérivée du ça, comme l'est aussi le surmoi qui présuppose le moi $^{2}$. Par la suite, cependant, le moi peut se renforcer pour s'autonomiser partiellement du ça, avec lequel il doit toutefois toujours composer. Cela, dit Bernet avec Freud, suppose que le moi se fasse aimer du ça en "s'enrichissant de tous les objets autrefois aimés par le ça, ensuite perdus et maintenant réunis sous la personnalité du ça $»^{3}$; pour se satisfaire lui-même, le ça a alors intérêt à préserver le moi, qui ne lui est plus totalement asservi. Dans une très complexe dialectique du maître et de l'esclave, le ça se sert du moi mais finit par en avoir intensément besoin au point de lui devoir des égards...

Toutes ces analyses constituent l'arrière-fond théorique à partir duquel Bernet relit les thèses husserliennes en matière de phénoménologie des actes pratiques. En insistant, avec les Ideen $I^{4}$, sur l'inséparabilité de la Seele (qui n'est dès lors pas seulement Geist) et du Leib (qui n'est dès lors pas seulement Körper $)^{5}$, Bernet fait valoir, d'une part, l'insuffisance du corps pour l'activité intentionnelle - en particulier, le corps sent mais ne perçoit pas, car seule l'âme est capable d'appréhensions intentionnelles ${ }^{6}$ - mais aussi, d'autre part, en sens inverse, toutes les conditionnalités psychophysiques (charnelles), idiopsychiques (liées aux expériences passées et à l'habituation) et intersubjectives (liées à l'environnement social) pesant sur l'activité de l'âme ${ }^{7}$. Loin d'être pleinement autonome, le pouvoir être subjectif, qui régit ses dispositions réactives, dépend fortement de ces conditionnalités qui le déterminent en tant que corps, en tant que corps doté d'une expérience passée et en tant que corps soumis à des contraintes sociales ${ }^{8}$. Dans les Études sur la structure de la conscience $^{9}$, rédigées à la même époque, Husserl s'intéresse plus précisément en-

\footnotetext{
${ }^{1}$ Ibid., p. 368-369.

${ }^{2}$ Ibid., p. 368.

${ }^{3}$ Ibid., p. 365.

${ }^{4}$ E. Husserl, Idées directrices pour une phénoménologie II, trad. fr. É. Escoubas, Paris, PUF, 1982.

${ }^{5}$ R. Bernet, Force - pulsion - désir, op. cit., p. 299-300.

${ }^{6}$ Ibid., p. 300-301.

${ }^{7}$ Ibid., p. 307-308.

${ }^{8}$ Ibid., p. 308.

${ }^{9}$ E. Husserl, Studien zur Struktur des Bewusstseins, édition critique à paraître dans les Husserliana.
} 
core aux rapports des actions (intentionnelles) du moi aux mouvements pulsionnels qui jaillissent de sa nature corporelle ${ }^{1}$. Comme les auteurs précédents, Husserl distingue les besoins purement corporels de ces tensions psychiques, étayées sur elles, que sont les pulsions. Comme les auteurs précédents, Husserl estime que les stimuli physiques qui déclenchent l'activation de ces pulsions en constituent moins la cause que l'occasion. Bien plus, comme Schopenhauer et Freud, Husserl s'intéresse au rôle des " motifs " pour transformer une pure énergétique pulsionnelle en activité rationnelle ${ }^{2}$. Husserl, en effet, distingue très nettement pulsion et souhait intentionnel orienté vers un objet, ainsi que plaisir sensible et plaisir de l'agrément ${ }^{3}$, lequel se rapporte à un objet pourvu de valeur intentionnel ${ }^{4}$. Entre pulsion et souhait, il envisage toutefois le niveau intermédiaire du désir, lequel n'est plus simple tension pulsionnelle, mais aspiration pulsionnelle au plaisir associé à la possession d'un objet singulier, et n'est pas encore vouloir intentionnel, mais seulement motivation de ce vouloir par la représentation d'un bien qui nous manque ${ }^{5}$. Chaînon manquant entre pulsionnalité et intentionnalité, le désir ainsi conçu se situe dans l'entrelacs de l'ordre des causes et celui des raisons ; suscité par les tensions pulsionnelles, il fournit au moi des « raisons » de le satisfaire, quoique pas encore tout à fait des justifications susceptibles de fonder une décision parfaitement rationnelle de la part du moi. Dans les mots de Bernet :

La première tâche d'une doctrine phénoménologique de la pulsion consiste tout naturellement à distinguer le faire et le laisser faire pulsionnels d'un agir actif. Une véritable action ou un agir actif repose, d'après Husserl, sur une décision volontaire du moi (un «fiat»), décision qui est rationnellement motivée par l'évidence de la possibilité pratique de l'action et par une conscience de sa valeur. [...] Cette distinction entre une soumission passive à la pulsion et un agir actif ne doit cependant pas donner à penser que les deux resteraient strictement séparés dans la vie concrète du sujet humain. Si tout agir rationnel procède de la motivation par un intérêt délibéré [dans ce cas, nous parlerons pour notre part de justification rationnelle], celui-ci peut cependant encore reposer sur une aspiration pulsionnelle [un désir] — à condition que cette « impulsion pulsionnelle » fasse l'objet d'un examen attentif de la part du moi délibérant. Jugeant du caractère rationnel ou irrationnel de la motivation pulsionnelle, le moi agissant lui « cède » ou au contraire la rejette. Comme Husserl accorde une grande

\footnotetext{
${ }^{1}$ R. Bernet, Force - pulsion - désir, op. cit., p. 314-315.

2 Ibid., p. 316-318.

${ }^{3}$ Ibid., p. 328.

${ }^{4}$ Ibid., p. 326.

${ }^{5}$ Ibid., p. 323.
} 
importance à cet examen actif des impulsions pulsionnelles passives, il consacre des recherches approfondies à la «promotion » (Förderung) ou au contraire à « l'inhibition » de celles-ci par le moi. Il mentionne explicitement, dans ce contexte, le «refoulement» et le «renoncement» (Entsagen) comme formes possibles d'une telle inhibition de la pulsion par le moi ${ }^{1}$.

\section{Les soubassements énergétiques de la perception}

Dans cette longue citation de Bernet commentant Husserl, on retrouve, dans la sphère de l'action pratique, tous les éléments que nous nous étions nous-même efforcé de faire valoir dans la sphère de l'activité théorique : au-delà des seules causes psycho-physiques mais en deçà des justifications rationnelles, les synthèses passives qui s'opèrent au sein même de l'expérience et indépendamment de l'activité subjective intentionnelle motivent les prises de position judicatives du moi en «l'inclinant à » ou en « lui fournissant des raisons de » donner son assentiment à ses synthèses. Si son activité intentionnelle est ainsi motivée et non purement arbitraire, le moi n'est toutefois pas déterminé par ces motifs ; il reste libre de les examiner et de les concéder ou de les rejeter, c'est-à-dire de désinhiber ou au contraire d'inhiber leur activation proprement intentionnelle.

Or, si l'analogie peut être maintenue entre la sphère affective et volitive et la sphère cognitive, cela veut dire qu'on pourrait aussi récupérer pas mal des analyses qu'a effectuées Bernet à partir d'Aristote, Leibniz, Schopenhauer et Freud, pour montrer comment ce niveau motivationnel émerge d'un pur jeu de forces organiques et de tensions psychiques, mais aussi comment la subjectivité elle-même à qui ces motivations s'adressent naît de ce même jeu. Or, que cette analogie (entre sphères volitive et cognitive) vaille, c'est ce que Bernet lui-même suggère en s'appuyant, il est vrai, sur une théorie phénoménologique de la connaissance, et notamment de la connaissance perceptive, qui est sans doute, plus que celle de Husserl, sensible à la continuité de l'activité subjective avec les processus psychophysiques qui lui sont sous-jacents, à savoir la théorie de la connaissance merleau-pontienne :

Étant donné que toute perception d'une chose par un sujet particulier émerge d'un « champ de perception » ou d'une « chair du monde » qui est parcouru par

${ }^{1}$ Ibid., p. 316-317. 
une multitude de forces qui circulent entre des éléments ressemblants et différents, proches et lointains, superficiels et profondément enfouis, il faut donc conclure que la perception est un processus qui a un caractère pulsionnel ${ }^{1}$.

Que, comme la volition, la perception soit un processus pulsionnel, cela voudrait alors dire que :

1) elle repose sur des processus dynamiques de nature purement organique - tensions et décharges énergétiques (activité neuronale), résistance exercée par la matière (la structure du système nerveux, telle que l'a notamment informée l'activité antérieure) ;

2) pour autant, la perception ne se réduit pas à ces processus dynamiques de nature purement organique, dans la mesure où elle ne prend une valeur proprement psychique que du fait d'être consciente ; comme la pulsion freudienne, la sensation est le représentant psychique des forces organiques ;

Une telle intervention de la conscience égoïque dans la réalisation d'une impulsion pulsionnelle n'est cependant concevable que si la pulsion est un phénomène psychique et non un pur phénomène physiologique ${ }^{2}$.

3) bien qu'elles soient généralement occasionnées par des stimuli extérieurs, par le «contact» avec des réalités externes - que Leibniz nommait analogiquement «perception»-, les sensations sont, comme les pulsions, déjà internes au mental et relèvent en ce sens de l'auto-affection - c'est là ce que pensait déjà Hume sous la notion d' « impression »; à l'égard de ces sensations, les stimuli constituent moins proprement des causes que des occasions ;

4) psychiques et non purement physiques, les sensations ne sont pas pour autant encore des attitudes intentionnelles ; elles constituent encore essentiellement des tendances (psychiques) à certains effets mentaux ou comportementaux ;

5) toutefois, les sensations ne mènent pas nécessairement à ces effets ; quoiqu'elles soient de l'ordre de la virtualité et poussent à s'actualiser dans la vie intentionnelle, elles peuvent se heurter à des inhibitions (dues à la résistance de la matière ou à d'autres forces, notamment sensorielles, en sens contraire) ;

6) en tant que forces psychiques confrontées à d'autres forces psychiques, les sensations ne sont pas totalement isolées des affects qui participent

${ }^{1}$ Ibid., p. 416.

${ }^{2}$ Ibid., p. 317. 
de la dynamique mentale d'origine corporelle ; le fait même que, par leur valeur affective, certaines sensations suscitent plus que d'autres l'intérêt et attirent plus que d'autres l'attention de l'esprit témoigne de ce que l'activité cognitive est fortement entremêlée à la dynamique affective et volitive ; des affects peuvent aussi jouer un rôle dans l'inhibition ou la désinhibition des tendances des sensations à s'actualiser en attitudes perceptives intentionnelles ;

7) dans cette possibilité qu'elles soient inhibées ou, au contraire, désinhibées par l'intervention d'autres forces ou éventuellement de représentations agissant à titre de "motifs », se joue le premier espace de liberté (au sens de non-détermination) et de rationalité de l'esprit — c'est la dunamis meta logou d'Aristote, intimement liée à une possible inhibition de l'actualisation de ce qui est en puissance, que Bernet qualifie joliment de « retenue dans l'exercice du pouvoir $\gg$;

8) le moi naît de ce jeu de forces et de cette indétermination de leur résolution ; comme le suggérait Leibniz, il n'est au fond que ce jeu de forces dans la mesure où il tend à s'unifier en un seul point de vue cohérent (raison pour laquelle on peut le lire comme visant à concilier les forces en présence, donc par exemple à laisser s'actualiser les sensations qui sont renforcées par d'autres et au contraire à affaiblir les sensations qui se heurteraient à beaucoup de forces ou motifs en sens contraire) ;

9) le moi semble toutefois une instance psychique dérivée de la dynamique et qui, comme l'indiquait Schopenhauer, permet seulement à cette dynamique de s'exercer de manière plus harmonieuse à long terme ;

10) en particulier, l'intérêt théorique que satisfait l'activité cognitive rationnelle peut être envisagé comme une ruse des intérêts pratiques pour optimiser leur satisfaction sur le long terme ;

11) dans la mesure, toutefois, où il joue son rôle régulateur, le moi gagne progressivement une certaine autonomisation à l'égard de la simple pulsionnalité sensorielle et affective.

Ce ne sont là, bien sûr, que les grandes lignes d'une esquisse de ce qui pourrait être étudié en détail sur le plan de la psychologie explicative à titre de soubassements génétiques de l'activité cognitive qui fait proprement l'objet de la psychologie descriptive et de la phénoménologie. Mais il y a là assurément un début d'explication cohérente à la genèse naturelle (pré-subjective) de la liberté et de la raison. 